\title{
Ciclo de Mejora en la enseñanza del inglés en la asignatura Idioma Moderno II
}

\section{An Improvement Cycle in English teaching within the subject of Idioma Moderno II}

Elisa díaz PRada

ORCID: https://orcid.org/0000-0003-2207-2489

Universidad de Sevilla

Departamento de Lengua Inglesa

ediazprada@us.es

DOI: http://dx.doi.org/10.12795/9788447231003.015

Pp.: 313-332 


\section{Contexto}

Idioma Moderno II (inglés) es una asignatura transversal anual de 12 créditos que pueden cursar alumnos pertenecientes a cualquiera de los grados que se imparten en la Facultad de Filología lo que implica que es un alumnado diverso, con distintas motivaciones y niveles en lengua inglesa.

Para cursar Idioma Moderno II se requiere disponer del libro del estudiante (New English File Upper-Intermediate) que es el manual que se ha venido usando desde hace años.

Por experiencias anteriores de mis compañeras, a mitad de curso, o incluso antes, solamente asisten a clase una media de 8-10 alumnos (de un total de unos 40-50 alumnos por clase). Esto se debe a que un gran número ya posee el nivel de inglés B2 (nivel que se exige haber alcanzado al finalizar la asignatura) y simplemente cursan esta asignatura por los créditos.

\section{Diseño previo del CIMA}

Este CIMA se ha diseñado para 8 horas de clase que equivalen a 6 sesiones (1h y 20 minutos cada una). El objetivo principal es que los alumnos tomen conciencia de los aspectos lingüísticos necesarios para narrar una historia o anécdota en inglés sobre un viaje real o imaginario. Para ello, se han tomado como referencia las unidades $2 \mathrm{~B}, 3 \mathrm{~A}$ y 3B del libro del estudiante de la asignatura, seleccionando aquellos contenidos que interesaban para el diseño del Ciclo de Mejora y descartando los que no eran necesarios. Se pueden ver ejemplos de estos contenidos en el mapa representado en la Figura 1, como Aprender a usar adverbios de tiempo, lugar, modo y opinión en una oración y un texto escrito. Cabe mencionar que no todos los contenidos provienen del libro sino que se han añadido otros como Prestar atención y cuidado a la presentación escrita que

Ciclos de Mejora en el Aula (2020). Experiencias de Innovación Docente de la US Esta obra se distribuye con la licencia Creative Commons 
se han considerado igualmente necesarios para conseguir la meta: narra una historia o anécdota sobre un viaje en inglés.

Todos estos temas representados en el mapa de contenidos se plantean para ser trabajados bajo cuatro grandes problemas que actúan de hilo conductor:

P1 ¿Qué función tienen los adjetivos en nuestra narración y cómo podemos usarlos?

P2 ¿Qué función tienen los adverbios en nuestra narración y cómo podemos usarlos?

P3 ¿Qué vocabulario podemos utilizar en nuestra historia?

P4 ¿Qué estructuras gramaticales debemos usar para narrar nuestra historia?

A su vez, estas cuatro cuestiones responden a la gran pregunta de ¿Qué recursos lingüísticos necesitamos para narrar nuestra historia?

Ciclos de Mejora en el Aula (2020). Experiencias de Innovación Docente de la US Esta obra se distribuye con la licencia Creative Commons 


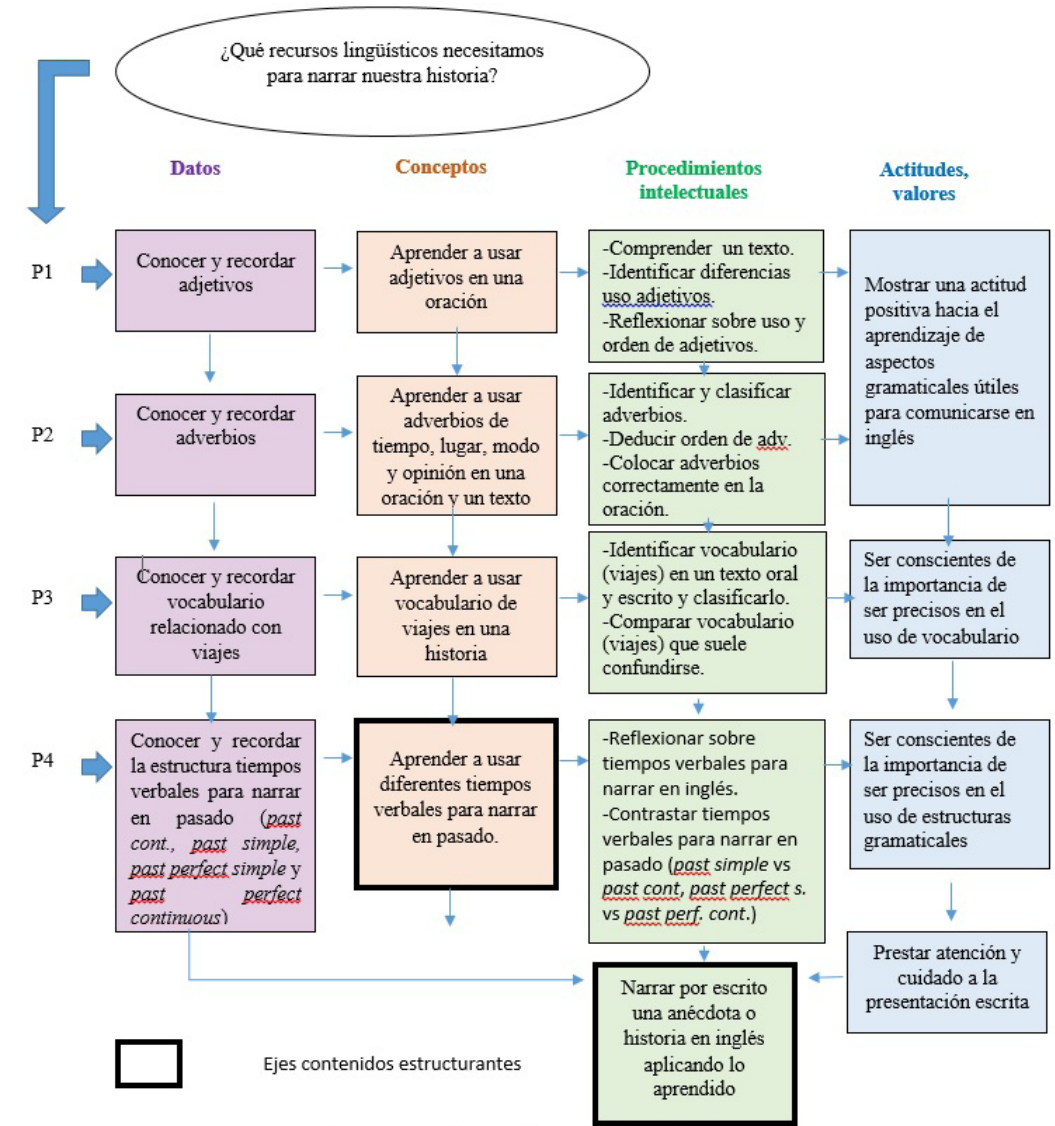

Figura 1. Mapa de contenidos y problemas clave

En el mapa de contenidos anterior se puede observar a la izquierda como cada uno de los problemas mencionados arriba da paso a cuatro tipos de contenidos diferenciados en colores: datos (morado), conceptos (naranja), procedimientos intelectuales (verde) y por último actitudes y valores (azules). Todos estos contenidos están a su vez relacionados entre sí puesto que cada contenido complementa al siguiente y todos juntos conforman un todo para poder narrar una historia

Ciclos de Mejora en el Aula (2020). Experiencias de Innovación Docente de la US Esta obra se distribuye con la licencia Creative Commons 
Para que los alumnos aprendan esos contenidos, es necesario que como docentes pensemos en la mejor forma de trabajarlos con nuestros estudiantes, es decir, qué método creemos que es el mejor, y a su vez posible para poder llevarlo a cabo.

Para una clase de lengua inglesa se diseña el siguiente modelo metodológico posible que consta de una serie de fases (cada círculo es una fase diferente).

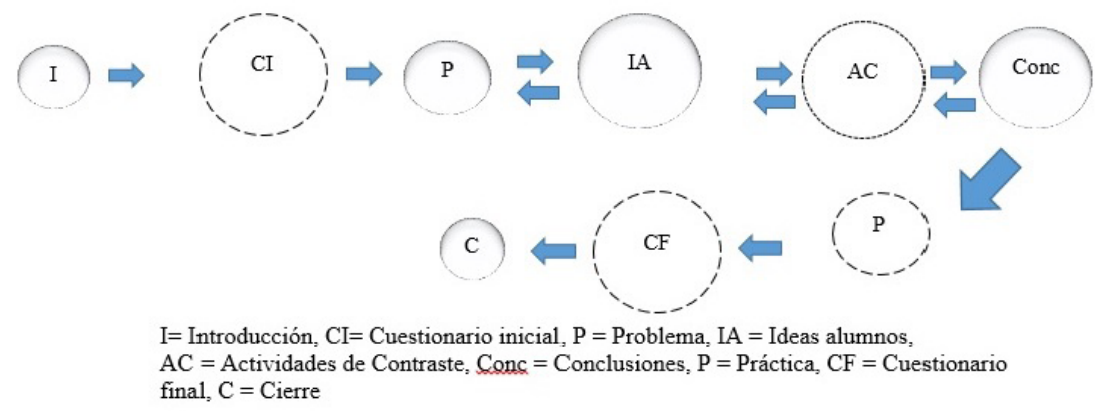

Figura 2. Modelo Metodológico posible

Los círculos con líneas discontinuas representan aquellas fases que no siempre tienen lugar a lo largo de las sesiones, sino una única vez (línea discontinua más alargada) o varias pero de forma intermitente (línea discontinua más corta).

Por tanto, si tenemos en cuenta aquellas fases que están siempre presentes y la fase $A C$ que es frecuente, nos encontramos con 6 fases predominantes cuyo núcleo lo conforman cuatro de ellas: el Problema formulado (P), las Ideas previas de los Alumnos (IA) seguidas de las Actividades de Contraste (AC), las cuales confrontan las ideas previas de los alumnos. Estas actividades culminan con la fase de Conclusiones (Conc) a la que los estudiantes llegan como grupo y/o de forma individual. Estas etapas tienen lugar tantas veces como sea necesario hasta que se considera que los alumnos han cambiado sus modelos mentales.

Ciclos de Mejora en el Aula (2020). Experiencias de Innovación Docente de la US Esta obra se distribuye con la licencia Creative Commons 
A su vez, cada ciclo incluye una o varias actividades y todas ellas forman la secuencia de actividades.

\section{Secuencia de actividades programadas}

A continuación se presenta una muestra de las secuencias de actividades programadas para una sesión, en concreto la quinta, donde se pueden observar las distintas fases a las que pertenecen así como el Problema que ejerce de hilo conductor de la sesión (P4 ¿Qué estructuras gramaticales debemos usar para narrar una historia?)

Tabla 1. Muestra de secuencia de actividades

Fase 1. Introducción

Act. 1

Saludo y objetivos

$1^{\prime}$

Saludo a los alumnos

Recapitulo brevemente lo visto en la clase anterior y lo enlazo con los objetivos de la sesión para que los alumnos sepan qué esperar y se preparen mentalmente.

Fase 2. Planteamiento del problema

Act. 2 Formulación problema

Tras haber trabajado otros elementos lingüísticos necesarios para narrar una anécdota, se les formula a los alumnos la siguiente pregunta para que reflexionen sobre los tiempos verbales: ¿Qué estructuras gramaticales debemos usar para narrar una historia?

Fase 3. Ideas de los Alumnos

Act. 3 Respuesta grupal $2^{\prime}$

Esta pregunta ya la reflexionaron de forma individual durante el cuestionario de la primera sesión. Por tanto, se forman grupos (de forma aleatoria en Blackboard Collaborate) y discuten las respuestas, las escriben en Wooclap. No corregimos todavía (se autocorregirán a medida que hagan la próxima actividad).

Recursos: Blackboard Collaborate, Wooclap.

Fase 4. Actividades de Contraste

Act. 4 Identificación de tiempos verbales

$15^{\prime}$

Ciclos de Mejora en el Aula (2020). Experiencias de Innovación Docente de la US Esta obra se distribuye con la licencia Creative Commons Reconocimiento-NoComercial-SinObraDerivada Internacional (CC BY-NC-ND 4.0.) 
Leen una historia breve e identifican los tiempos verbales usados.

Contrastan los tiempos encontrados con sus respuestas anteriores y se hace una puesta en común con toda la clase.

Recursos: archivo subido a Enseñanza Virtual

Fase 5. Conclusiones

Act. 5 Deducción y conclusiones

Con la reflexión anterior y la corrección de la actividad de contraste, los propios alumnos llegan a sus propias conclusiones. Si fuera necesario, la profesora añade información.

\section{Cuestionario inicial-final}

Con el objetivo de hacer un seguimiento de la evolución de los estudiantes en relación con los problemas y los contenidos planteados, se diseña un cuestionario que los alumnos responden al comienzo de la primera sesión y al final de la última. El contenido de ambos cuestionarios es idéntico y se estructura en torno a varias preguntas que se les van formulando a los alumnos y que responden a su vez a los cuatro grandes problemas mencionados anteriormente. A continuación se presenta dicho cuestionario.

1. Lee estas descripciones sacadas de anuncios ¿Cuáles te llaman más la atención? ¿Qué productos/servicios consumirías? ¿Por qué?

- New apartment in the city centre. Kitchen includes furniture and appliances. Big bathroom.

- Leather jacket of good quality.

- Experienced and certified native teacher offers enjoyable and carefully designed English language classes.

2. ¿Crees que el lenguaje usado ha influido en tu decisión? ¿Por qué?

3. Compara estas descripciones de los anuncios con las anteriores ¿Qué diferencias observáis? ¿Qué anuncios elegiríais ahora? ¿Por qué? ¿Ha cambiado vuestra elección anterior?

Ciclos de Mejora en el Aula (2020). Experiencias de Innovación Docente de la US Esta obra se distribuye con la licencia Creative Commons 


\section{Apartment}

- New apartment in the city centre. Kitchen includes furniture and appliances. Big bathroom.

- Magnificient newly built apartment in the heart of Seville, fully and furnished and equipped kitchen, beautiful spacious bathroom.

\section{Lacket}

- Leather jacket of good quality.

- Stunning looking dark navy leather jacket. Excellent quality.

\section{English classes}

- Experienced and certified native teacher offers enjoyable and carefully designed English language classes,

- Native teacher with experience. Certification available. Classes are well prepared and ejoyable.

4. Observa detenidamente la posición de los adjetivos subrayados en las frases anteriores y responde a las preguntas.

\section{beautiful spacious bathroom.}

¿Por qué beautiful va antes que spacious?

stunning looking dark navy leather jacket

¿Por qué stunning looking va antes que dark navy?

¿Por qué dark navy va antes que leather?

enjoyable and carefully designed English language classes,

- ¿Por qué enjoyable va antes que carefully designed?

- ¿Por qué carefully designed va antes que English language?

- ¿Qué orden crees que deben seguir los adjetivos antes del sustantivo?

Ciclos de Mejora en el Aula (2020). Experiencias de Innovación Docente de la US Esta obra se distribuye con la licencia Creative Commons 
5. Observa las imágenes y lee los textos que las acompañan. ¿Qué diferencia común hay entre los textos debajo de cada imagen? Es la misma diferencia para los cuatro textos.

[Incluyo sólo uno de los textos por límite de espacio]

'What are you reading there? It looks serious - you must be incredibly smart.' He uses his usual chat-up lines on the train. Ask them a simple questions. Then pay them a compliment. It always works with women. Sadly not this time.

'What are you reading there? It looks serious - you must be smart.' He uses his usual chat-up lines on the train. Ask them a simple questions. Then pay them a compliment. It works with women. Not this time.

6. ¿Cómo clasificarías los adverbios subrayados? Pon un nombre a cada categoría.

[Incluyo sólo un extracto por límite de espacio]

'What are you reading there? It looks serious - you must be incredibly smart.' He uses his usual chat-up lines on the train. Ask them a simple questions. Then pay them a compliment. It always works with women. Sadly not this time.

7. ¿Cuál crees que es la norma a seguir para la posición de los distintos tipos adverbios que acabas de clasificar en una oración? (responde por categorías no adverbio por adverbio)

8. De las diferentes categorías que has establecido ¿cuál/es piensas que sería/n más apropiados para la narración de una anécdota?

9. ¿Qué vocabulario relacionado con viajes se podría usar en vuestras narraciones? ¿Cómo lo clasificarías por categorías?

10. ¿Cuál es la diferencia entre travel, trip y journey?

Ciclos de Mejora en el Aula (2020). Experiencias de Innovación Docente de la US Esta obra se distribuye con la licencia Creative Commons 
11. ¿Qué tiempos verbales necesitamos para narrar una anécdota?

12. ¿Cuáles crees que son las diferencias entre el past simple y el past continuous? ¿y entre el past perfect simple y past perfect continuous?

\section{Aplicación del CIMA}

En este apartado se incluye por una parte una descripción de las sesiones y por otra, la evaluación del aprendizaje de los estudiantes tras las 8 horas de la aplicación del Ciclo de Mejora.

\section{Desarrollo de las sesiones}

En general, las sesiones transcurrieron con normalidad, con la sensación de que lo planificado y el modelo metodológico funcionaban. Surgieron algunos aspectos que merece la pena mencionar para la aplicación de un futuro CIMA. Los describo brevemente a continuación:

- En la Sesión 1 (centrada en el uso de los adjetivos) hubo alumnos que no entendieron la segunda parte de uno de los problemas planteados (se subraya):

Lee estas descripciones sacadas de anuncios ¿Cuáles te llaman más la atención? ¿Qué productos/servicios consumirías? ¿Por qué? Quizás debería haber usado un lenguaje más sencillo. En lugar de ¿Qué productos/servicios consumirías? preguntar ¿Te alquilarías el piso? ¿Te comprarías la chaqueta?, etc.

- No fue hasta poco antes de la Sesión 3 cuándo me di cuenta de que estaba poniendo como actividades prácticas unas actividades que en realidad eran de contraste. Además, estas actividades "prácticas" las estaba incluyendo en la penúltima fase, cuando deberían ir

Ciclos de Mejora en el Aula (2020). Experiencias de Innovación Docente de la US Esta obra se distribuye con la licencia Creative Commons 
acompañando a cada ciclo de problema-ideas alumnos-conclusiones. No tiene sentido hacerles reflexionar sobre algo y luego no aportarles actividades para que vayan modificando sus ideas. Realicé las modificaciones oportunas a partir de la Sesión 4.

- El programa de Wooclap que usé en muchas de las sesiones para el planteamiento de problemas tuvo una gran acogida por parte de los alumnos. Esta herramienta permite al profesor proyectar una o varias preguntas y visualizar las respuestas de los alumnos a medida que las van escribiendo. Su éxito reside en que permite al docente presentar los problemas de una forma visual y motivadora.

- La falta de tiempo, aunque tuvo lugar en alguna que otra clase, fue la protagonista en las últimas sesiones. Durante la Sesión 5, los alumnos se "atascaron" en una de las actividades de contraste relacionadas con las diferencias entre los distintos tiempos verbales, lo que implicó asignarles la tarea para casa. La Sesión 6 comenzó, por tanto, con la corrección de esa actividad que una vez más llevó más tiempo del esperado. Los alumnos tenían dudas y fue necesario parar en cada punto. Como consecuencia, la práctica escrita (narración de la historia) se tuvo que asignar como tarea para casa, explicando en detalle las instrucciones y facilitándoles unas directrices por escrito. Era necesario dejar los 20 minutos restantes para que los alumnos contestaran el cuestionario final y así cerrar el CIMA. El tiempo establecido para el mismo fueron 10 minutos menos que el dado para realizar el cuestionario inicial. Supuse que al conocer ya las preguntas y en teoría las respuestas, a los estudiantes les iba a resultar más fácil e iban a contestar rápido. Sin embargo, no fue así ya que algunos no terminaron el cuestionario y otros lo contestaron con prisa.

Esta experiencia ha tenido una doble repercusión. Por una parte, se ha convertido en el impulso necesario para continuar y diseñar un futuro CIMA. Por otra, ha servido

Ciclos de Mejora en el Aula (2020). Experiencias de Innovación Docente de la US Esta obra se distribuye con la licencia Creative Commons 
para realizar algunas mejoras en la planificación, como por ejemplo reducir el contenido y aumentar el tiempo establecido para las actividades, especialmente aquellas que merecen una especial atención, ya sea la reflexión de los alumnos, una actividad de contraste o el cuestionario. De hecho, mi compañero con el que realicé las observaciones cruzadas menciona lo siguiente: Como único aspecto mejorable, considero que sería útil delimitar el tiempo de las actividades que ha diseñado, pues ello puede hacer que en ocasiones pueda dejar alguna tarea sin concluir. Esta sería una cuestión de ajustar simplemente el tiempo y no excederse en el diseño de tareas que puedan quitarle tiempo a otras actividades más esenciales para la resolución del problema principal.

\section{Evaluación del aprendizaje}

En segundo lugar, para poder evaluar el porcentaje de mejora de los alumnos se ha procedido a comparar los conocimientos iniciales de 12 alumnos con los finales, tomando como referencia las respuestas dadas en el cuestionario inicial y final. Una vez analizadas y contrastadas, se identificaron distintos "modelos mentales" de los alumnos y se representaron en una "Escalera de Aprendizaje" (Porlán, 2017, p. 81). Se ha diseñado una escalera por cada pregunta con lo cual se han obtenido 12. Sin embargo, por limite de espacio se presentan a continuación solamente tres muestras representativas.

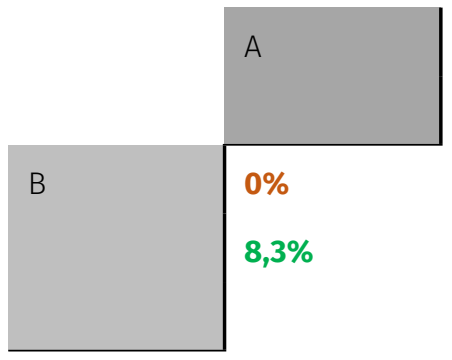

Ciclos de Mejora en el Aula (2020). Experiencias de Innovación Docente de la US Esta obra se distribuye con la licencia Creative Commons 


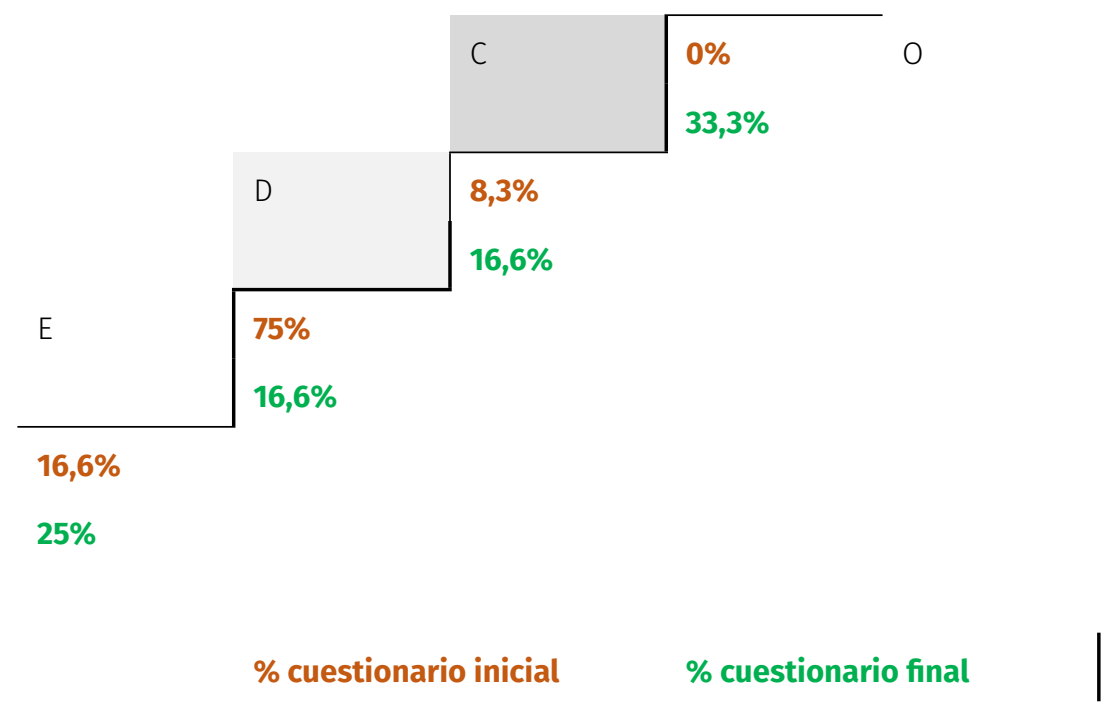

Figura 3. Escalera de aprendizaje de la Pregunta 4: Observa detenidamente la posición de los adjetivos en negrita en las frases anteriores y responde a las preguntas.

Modelo A. Los estudiantes justifican correctamente la posición de los adjetivos en los ejemplos ofrecidos y conocen el orden que deben seguir antes del sustantivo.

Modelo B. Los estudiantes justifican parcialmente, o no conocen, la posición de los adjetivos en los ejemplos ofrecidos, pero conocen el orden que deben seguir antes del sustantivo.

Modelo C. Los estudiantes justifican parcialmente la posición de los adjetivos en los ejemplos ofrecidos y conocen parcialmente el orden que deben seguir antes del sustantivo.

Modelo D. Los estudiantes no justifican de forma adecuada la posición de los adjetivos en los ejemplos ofrecidos ni conocen el orden que deben seguir antes del sustantivo.

Modelo E. No contesta/ no procede

Obstáculo (O). No son conscientes de que hay una norma para el orden de los adjetivos.

Ciclos de Mejora en el Aula (2020). Experiencias de Innovación Docente de la US Esta obra se distribuye con la licencia Creative Commons 


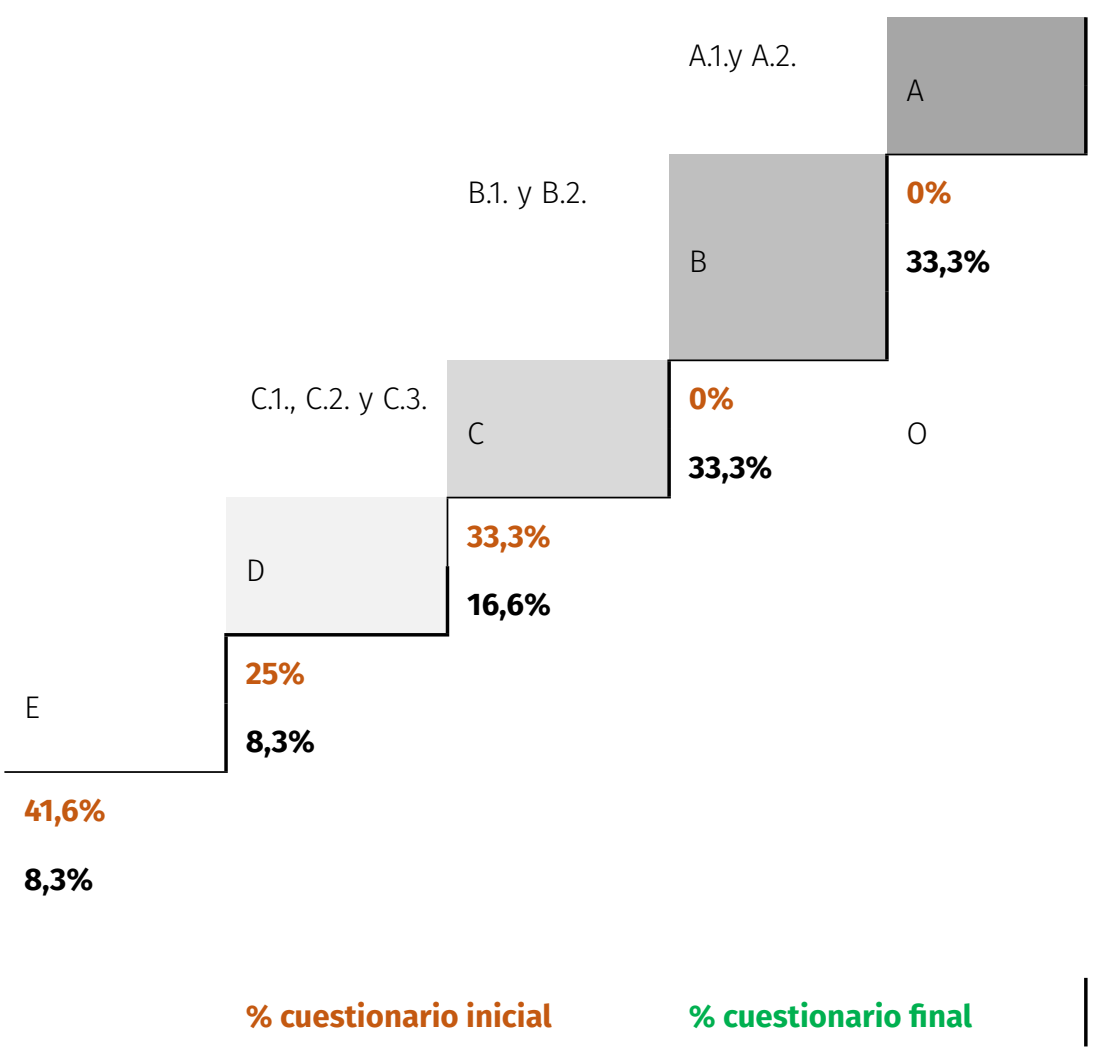

Figura 4. Escalera de aprendizaje de la Pregunta 6: ¿Cómo clasificarías los adverbios resaltados en negrita en el texto? Pon un nombre a cada categoría.

Modelo A. Los estudiantes presentan las 5 categorías correctas para clasificar los adverbios (tiempo, frecuencia, modo, cantidad y opinión)

A.1.Incluye el $100 \%$ de ejemplos correctos.

A.2. Incluye entre el 70-90\% de ejemplos correctos.

Modelo B. Los estudiantes presenta 4 categorías correctas para clasificar los adverbios (tiempo, modo, cantidad y de opinión).

B. 1. Incluye entre 50-60\% de ejemplos correctos.

B.2. No incluye ejemplos correctos.

Modelo C. Los estudiantes presentan 3 categorías correctas para clasificar los adverbios (principalmente: tiempo, modo y cantidad)

Ciclos de Mejora en el Aula (2020). Experiencias de Innovación Docente de la US Esta obra se distribuye con la licencia Creative Commons 
C.1. Incluye entre $60-75 \%$ de ejemplos correctos.

C.2. Incluye entre 50-60\% de ejemplos correctos.

C.3.No incluye ejemplos correctos de adverbios

Modelo D. El estudiante presentan 1-2 categorías correctas (tiempo, modo) para clasificar los adverbios e incluye el 25\% de ejemplos correctos de adverbios.

Modelo E. No contesta/no procede

Obstáculo (O). Desconocen o no recuerdan la tipología de adverbios. Clasifican adverbios en la categoría incorrecta.

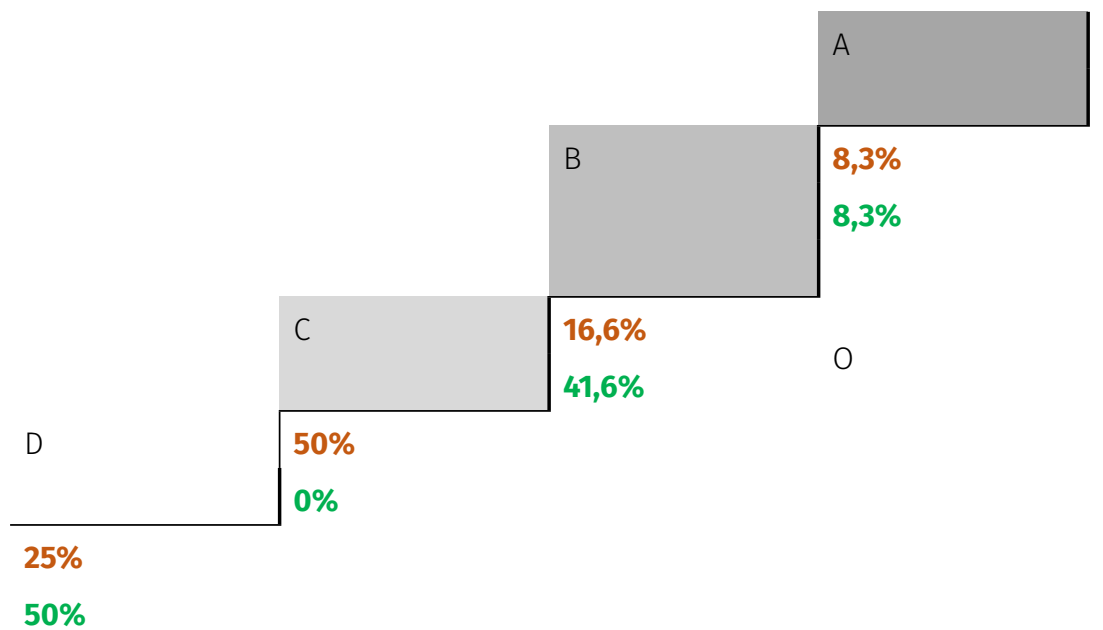

$\%$ cuestionario inicial

$\%$ cuestionario final

Figura 5. Escalera de aprendizaje de la Pregunta 10: ¿Cuál es la diferencia entre travel, trip y journey?

Modelo A. Los estudiantes diferencian perfectamente los términos travel, trip y journey.

Modelo B. Los estudiantes diferencian parcialmente los tres términos.

Modelo C. Los estudiantes no diferencian los tres términos.

Modelo D. No contesta/no procede

Ciclos de Mejora en el Aula (2020). Experiencias de Innovación Docente de la US Esta obra se distribuye con la licencia Creative Commons 
Obstáculo (0): Han usado indistintamente estas palabras y no se han planteado posibles diferencias de significado. Posible fosilización del uso de travel como sustantivo en lugar de trip.

Cada escalera corresponde a una pregunta de las planteadas en el cuestionario inicial-final (ver apartado "Cuestionario inicial-final"). En cada escalón aparecen representados cada uno de los modelos mentales de los estudiantes identificados con letras de la E o la D a la A, donde la A representa el modelo esperado y a medida que la escalera desciende los modelos se van alejando de lo esperado.

La altura de los escalones es diferente, siendo mayor cuando se estima que el "salto" de un modelo a otro puede resultar más difícil a los alumnos. Debajo de cada escalón se muestran los porcentajes de alumnos que han seguido ese modelo: en color naranja aparecen los resultados correspondientes al cuestionario inicial y en verde los relativos al final. Igualmente se describen los posibles obstáculos encontrados (O) que les impiden avanzar en la escalera, especialmente entre aquellos peldaños de mayor altura.

En el caso de la segunda escalera, se han añadido a la izquierda de cada peldaño una subclasificación de los modelos puesto que el problema formulado requería una doble respuesta. En las dos primeras escaleras, aunque más de la mitad de los alumnos no ha alcanzado el último escalón, llama la atención la diferencia entre las respuestas iniciales y las finales, en algunos casos pasando de $0 \%$ al $33 \%$ en los escalones más avanzados.

La tercera escalera contrasta con las dos anteriores puesto que, aunque es cierto que ha habido mejora y que el gran salto se produce entre el escalón C y el B (el de mayor altura), sólo el 8,3\% de los alumnos (es decir, un solo alumno o alumna) ha sido capaz de distinguir las diferencias entre los tres términos planteados en la pregunta. Por tanto, queda constancia de la dificultad de esta pregunta y de la necesidad de trabajarla más en clase. 


\section{ELISA DÍAz PRADA}

Finalmente, se incluye a continuación el cuadro de evaluación el que se pueden observar los porcentajes de mejora de cada uno de los 12 alumnos evaluados, resultados que se han calculado a partir de cada una de las escaleras. Los resultados destacados en azul representan una mejora en el cuestionario final en comparación con el inicial mientras que en rojo lo contrario. En la última columna se han representado los porcentajes totales de mejora por alumno.

\section{Tabla 2. Cuadro de evaluación}

\begin{tabular}{|c|c|c|c|c|c|c|c|c|c|c|c|c|c|c|c|c|c|c|c|c|c|c|c|c|c|c|c|c|c|c|c|c|c|c|c|c|c|}
\hline $\mathbf{E}$ & $1 \mathrm{i}$ & If & C & $2 \mathbf{i}$ & $2 f$ & C & $3 \mathbf{i}$ & $3 \mathrm{f}$ & C & $4 i$ & $\mathbf{4 f}$ & C & $5 \mathbf{i}$ & $5 f$ & $\mathbf{C}$ & $6 i$ & $6 \mathrm{f}$ & C & $7 \mathbf{i}$ & $7 \mathrm{f}$ & C & $8 i$ & $8 \mathrm{f}$ & C & $9 \mathrm{i}$ & 9f & C & $10 \mathrm{i}$ & $10 \mathrm{f}$ & C & $11 \mathrm{i}$ & $11 \mathrm{f}$ & C & $12 \mathrm{i}$ & $12 \mathrm{f}$ & C & $\mathbf{M}$ \\
\hline 1 & C & D & $-20 \%$ & D & E & $-20 \%$ & B & D & $-60 \%$ & E & E & $0 \%$ & B & C & $-40 \%$ & E & E & $0 \%$ & E & E & $0 \%$ & E & E & $0 \%$ & D & D & $0 \%$ & C & D & $-20 \%$ & B & D & $-60 \%$ & B & D & $-60 \%$ & $0 \%$ \\
\hline 2 & B & B & $0 \%$ & C & C & $0 \%$ & B & B & $0 \%$ & C & B & $40 \%$ & A & A & $0 \%$ & C & A & $60 \%$ & C & C & $0 \%$ & E & $\mathrm{B}$ & $0 \%$ & $R$ & A & $20 \%$ & B & B & $0 \%$ & A & A & $0 \%$ & B & A & $20 \%$ & $12 \%$ \\
\hline 3 & A & B & $-20 \%$ & $\mathrm{C}$ & B & $40 \%$ & B & B & $0 \%$ & D & D & $0 \%$ & A & A & $0 \%$ & D & A & $80 \%$ & C & B & $40 \%$ & c & B & $40 \%$ & C & A & $60 \%$ & $\mathrm{C}$ & B & $40 \%$ & A & A & $0 \%$ & B & B & $0 \%$ & $23 \%$ \\
\hline 4 & B & A & $20 \%$ & B & B & $0 \%$ & B & B & $0 \%$ & D & B & $60 \%$ & A & A & $0 \%$ & C & C & $0 \%$ & C & C & $0 \%$ & B & B & $0 \%$ & D & C & $20 \%$ & C & B & $40 \%$ & B & A & $20 \%$ & B & B & $0 \%$ & $13 \%$ \\
\hline 5 & $\mathrm{C}$ & $\mathrm{C}$ & $0 \%$ & D & D & $0 \%$ & C & A & $60 \%$ & D & C & $20 \%$ & B & A & $20 \%$ & E & C & $40 \%$ & D & D & $0 \%$ & E & B & $80 \%$ & D & A & $80 \%$ & D & D & $0 \%$ & D & D & $0 \%$ & D & D & $0 \%$ & $25 \%$ \\
\hline 6 & A & A & $0 \%$ & A & B & $-20 \%$ & B & B & $0 \%$ & D & A & $80 \%$ & B & A & $20 \%$ & D & B & $60 \%$ & 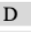 & C & $20 \%$ & C & A & $60 \%$ & D & B & $60 \%$ & B & D & $-60 \%$ & C & D & $-20 \%$ & B & D & $-60 \%$ & $12 \%$ \\
\hline 7 & C & C & $0 \%$ & D & B & $60 \%$ & A & A & $0 \%$ & D & B & $60 \%$ & B & A & $20 \%$ & E & A & $100 \%$ & C & A & $60 \%$ & B & A & $20 \%$ & C & B & $40 \%$ & C & A & $60 \%$ & C & B & $40 \%$ & D & C & $20 \%$ & $40 \%$ \\
\hline 8 & B & C & $-40 \%$ & B & D & $-60 \%$ & B & A & $20 \%$ & D & C & $20 \%$ & B & A & $20 \%$ & E & D & $20 \%$ & E & E & $0 \%$ & E & E & $0 \%$ & D & D & $0 \%$ & D & D & $0 \%$ & D & D & $0 \%$ & D & D & $0 \%$ & $0 \%$ \\
\hline 9 & C & A & $60 \%$ & B & B & $0 \%$ & B & B & $0 \%$ & D & D & $0 \%$ & B & B & $0 \%$ & E & B & $80 \%$ & E & E & $0 \%$ & E & E & $0 \%$ & D & $D$ & $0 \%$ & C & D & $-20 \%$ & B & D & $-60 \%$ & D & D & $0 \%$ & $5 \%$ \\
\hline 10 & B & B & $0 \%$ & D & B & $60 \%$ & B & B & $0 \%$ & E & E & $0 \%$ & A & A & $0 \%$ & C & B & $40 \%$ & C & C & $0 \%$ & E & E & $0 \%$ & D & C & $20 \%$ & D & B & $60 \%$ & D & A & $80 \%$ & D & B & $60 \%$ & $27 \%$ \\
\hline 11 & A & A & $0 \%$ & A & A & $0 \%$ & B & A & $20 \%$ & D & B & $60 \%$ & A & A & $0 \%$ & C & A & $60 \%$ & C & A & $60 \%$ & B & D & $-60 \%$ & D & B & $60 \%$ & C & D & $-20 \%$ & B & D & $-60 \%$ & C & D & $-20 \%$ & $8 \%$ \\
\hline 12 & C & $\mathrm{C}$ & $0 \%$ & D & D & $0 \%$ & B & B & $0 \%$ & D & $F$ & $-20 \%$ & B & A & $20 \%$ & D & B & $60 \%$ & 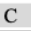 & B & $40 \%$ & 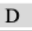 & 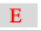 & $-20 \%$ & $D$ & 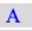 & $80 \%$ & A & B & $-20 \%$ & B & B & $0 \%$ & C & C & $0 \%$ & $12 \%$ \\
\hline
\end{tabular}

\section{E: Estudiante C: Cambio M: Mejora}

En esta tabla se presentan los modelos de los estudiantes (letras A, B, C, D y E) extraídos de cada una de sus respuestas aportadas a las 12 preguntas de los cuestionarios inicial (i) y final (f). La columna "C" que aparece a la derecha de cada pregunta nos muestra el cambio producido por cada alumno en el cuestionario final en relación al cuestionario inicial.

El color azul representa avances/saltos hacia arriba mientras que el color rojo representa un retroceso.

Ciclos de Mejora en el Aula (2020). Experiencias de Innovación Docente de la US

cc (i) $\odot$ Esta obra se distribuye con la licencia Creative Commons

(c) $(7) \Theta$ Reconocimiento-NoComercial-SinObraDerivada

4.0 Internacional (CC BY-NC-ND 4.0.) 
En la última columna se puede observar el porcentaje de mejora total de cada estudiante.

\section{Evaluación del CIMA}

Después de la aplicación del CIMA me planteo cuatro grandes cambios:

- Añadir más Actividades de Contraste para trabajar determinados contenidos que a los estudiantes les han resultado más difíciles, por ejemplo, la diferencia entre los distintos tiempos verbales para narrar una historia o entre los conceptos planteadas en la pregunta 10 (ver tercera escalera).

- Diseñar un futuro CIMA con menos contenidos y reducir el número de problemas a dos con sus correspondientes preguntas de enlace, sin que sobrepasen 6 preguntas en total, porque si no, los cuestionarios son largos e impiden evaluar en ocasiones de forma completa y fiable a un alumno que por falta de tiempo o por dejadez no contesta adecuadamente.

- Incorporar el modelo metodológico posible diseñado donde prevalezcan las fases de Problema, Actividades de Contraste, Ideas de los Alumnos y Conclusiones, dedicando más tiempo a las Ideas de los Alumnos y a las Actividades de Contraste.

- Aplicar la forma de evaluación por medio del cuestionario inicial-final e intentar plasmar de alguna forma el progreso del estudiante en la calificación.

- Son muchos los Principios Didácticos que como docente he ido incorporando a lo largo de mi carrera profesional. A continuación destaco aquellos que me han acompañado durante esta experiencia y que incorporo a mi Modelo Didáctico Personal:

- Centrar el aprendizaje en el alumno y hacerlo de forma auténtica. Para ello se trabajarán los distintos tipos de contenidos a partir de unos problemas planteados (no del libro de texto) que fomentarán la reflexión de

Ciclos de Mejora en el Aula (2020). Experiencias de Innovación Docente de la US Esta obra se distribuye con la licencia Creative Commons 
los estudiantes y ayudarán a crear un "entorno para el aprendizaje crítico natural” (Bain, 2007, p. 114)

- Seleccionar cuidadosamente los contenidos y planificar de forma exhaustiva la secuencia de actividades dedicando un tiempo mayor a aquellas actividades que requieren una mayor reflexión.

- Dar más importancia al aprendizaje progresivo, no a los resultados obtenidos en una única prueba final.

- Autoevaluarnos como docentes porque siempre se puede mejorar.

Por último, el diseño y aplicación del CIMA ha supuesto una gran dedicación de tiempo para planificar, estructurar y reflexionar, tiempo que se compensa con creces por los resultados obtenidos, especialmente un cambio de mentalidad como docente. Veo esta experiencia como el punto de partida hacia un gran cambio en mi docencia. He iniciado un camino de no retorno.

Ciclos de Mejora en el Aula (2020). Experiencias de Innovación Docente de la US Esta obra se distribuye con la licencia Creative Commons 
Palabras clave: Idioma Moderno II, inglés, Grados Facultad de Filología, Docencia Universitaria, Experimentación Docente Universitaria.

Keywords: Idioma Moderno II, English, Degree Courses at the Faculty of Philology, University teaching, University Teaching Experimentation.

\section{Referencias bibliográficas}

Bain, K. (2007). Lo que hacen los mejores profesores universitarios. Valencia: Universidad de Valencia

Porlán, R. (2017). Enseñanza universitaria. Cómo mejorarla. Madrid: Morata.

Ciclos de Mejora en el Aula (2020). Experiencias de Innovación Docente de la US Esta obra se distribuye con la licencia Creative Commons 